\title{
Radio observations of ultracompact HII regions
}

\author{
Luis F. Rodríguez \\ Centro de Radioastronomía y Astrofísica, UNAM, Apdo. Postal 3-72, Morelia, Michoacán \\ 58089 México \\ email: 1.rodriguez@astrosmo.unam.mx
}

\begin{abstract}
I review recent developments in the field of radio observations of ultracompact H II (UCHII) regions that have appeared in the literature since the last major review (Churchwell 2002). The morphology of UCHII regions continues to be a topic of research and recent studies of individual regions of star formation suggest modifications with respect to the results obtained from galactic plane surveys. I also briefly touch upon the question of the density structure of UCHII regions. Finally, I will also discuss the evidence of time variability in UCHII regions, and present examples of objects that are expanding, varying in flux density, or moving in the sky.
\end{abstract}

Keywords. Stars: formation, HII regions, radio continuum: ISM

\section{Introduction}

The ultracompact H II (UCHII) regions are small (with radius smaller than $10^{17} \mathrm{~cm}$ ), dense (with electron densities larger than $10^{4} \mathrm{~cm}^{-3}$ ) volumes of gas ionized by deeply embedded, young OB stars. Since the UCHII regions can be strong emitters of free-free continuum and radio recombination lines, they are an attractive target for observers and theoreticians alike. The large extinction present in the molecular cloud in which the UCHII regions are embedded generally limits the observations to the radio and infrared wavelengths. In this contribution I will emphasize recent developments obtained since the thorough review of Churchwell (2002), giving special attention to the existence of time variation in these compact objects.

\section{Morphology}

Churchwell (2002) classifies the morphology of UCHII regions in four categories: shelllike, cometary, core-halo, and bipolar (see Fig. 1), with the addition of spherical, unresolved, and irregular morphologies. The morphologies are important because they keep memory of the molecular surroundings of the young OB star, before ionization began. Of course, the present morphology is the result of the interplay of the original molecular morphology with the effects of ionization and of the powerful winds and outflows that are produced by massive stars.

In a recent paper, De Pree et al. (2005) studied with the Very Large Array more than 100 UCHII regions in Sgr B2 and W49A. Although they find similar morphologies than those found in the galactic plane surveys of Wood \& Churchwell (1989) and Kurtz et al. (1994), De Pree et al. (2005) note an important difference in their study.

De Pree et al. (2005) find a much larger percentage of shell-like UCHII regions (28\%) in comparison to the values of $4 \%$ and $1 \%$ found by Wood \& Churchwell (1989) and Kurtz et al. (1994), respectively. At present it is unclear if this discrepancy is due to special conditions in the two extreme star-forming regions studied by De Pree et al. (2005) or 

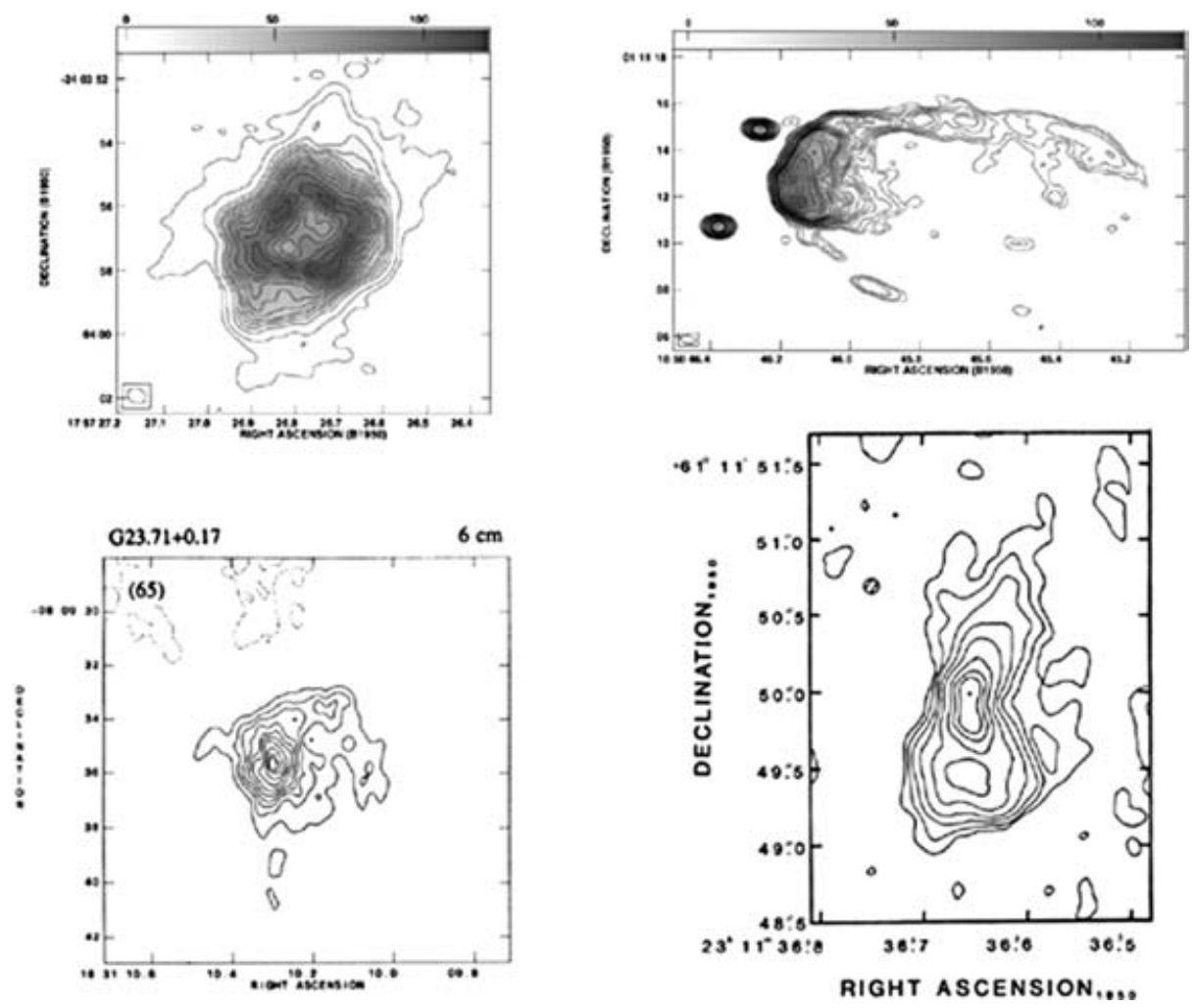

Figure 1. Basic morphologies of UCHII regions: shell-like (top left), core-halo (bottom left), cometary (top right), and bipolar (bottom right). From Churchwell (2002).

to differences in the observational techniques. Brief observations called snapshots are typically used in the surveys while more detailed imaging with better coverage of the $(\mathrm{u}, \mathrm{v})$ plane can be achieved when a few selected regions are being studied. The latter approach may lead to a better imaging of the sources and may favor the "discovery" of shell-like morphologies. It is interesting to point out that the detailed VLA study of NGC 6334 by Carral et al. (2002) also shows a large fraction of shell-like H II regions.

DePree et al. (2005) also propose to eliminate the core-halo morphology since they argue that essentially all UCHII regions are associated with large-scale, diffuse emission, when observed with sufficient sensitivity (Kim \& Koo 2003). Consequently, the "corehalo" designation is not a useful morphological discriminant in modern data sets of high quality.

\section{Electron Density Structure}

It is frequent for ultracompact and hypercompact H II regions to exhibit a continuum spectrum that is a power law of the form $S_{\nu} \propto \nu^{1}$. Following Panagia \& Felli (1975) it is reasonable to interpret this power law spectrum as due to a power law in the electron density as a function of radius. Indeed, for a spherical ionized region where the electron density goes as

$$
n_{e} \propto r^{-\beta}
$$




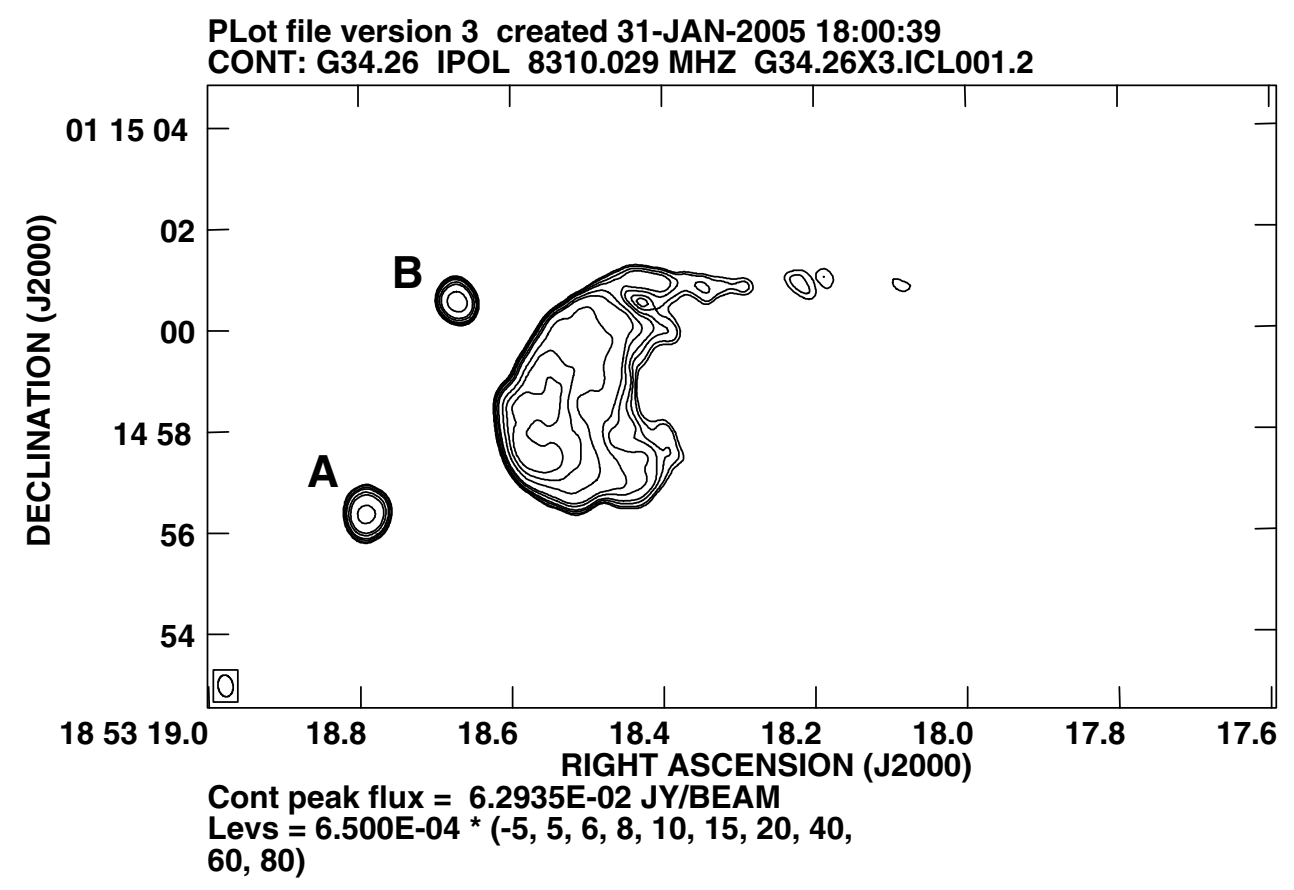

Figure 2. VLA 3.6-cm image (A configuration, ROBUST $=0$ ) of the G34.26+0.15 region, showing the two hypercompact H II regions A and B to the east of the cometary H II region.

with $r$ being the radius of the point considered, the flux density of the source goes as

$$
S_{\nu} \propto \nu^{(4 \beta-6.2) /(2 \beta-1)},
$$

while the angular size of the source goes as

$$
\theta_{\nu} \propto \nu^{-2.1 /(2 \beta-1)}
$$

Franco et al. (2000) have proposed that the explanation for the power laws of order $S_{\nu} \propto \nu^{1}$ can be obtained with a dependence of the electron density of the form $n_{e} \propto r^{-2.6}$. However, following the formulae listed above, we expect the angular size of the region to go with frequency as $\theta_{\nu} \propto \nu^{-0.5}$. This dependence of the angular size with frequency has not been tested observationally because most ultracompact and hypercompact H II regions are in complex fields where it is very hard to measure angular sizes accurately.

Avalos et al. (2005) have tried to measure this dependence of the angular size with frequency in the hypercompact H II regions A and B, that are associated with the cometary H II region G34.26+0.15 (see Fig. 2). Interestingly, they find that although the spectral indices are indeed found to be $\sim 1$, the angular sizes do not decrease significantly with frequency (see Fig. 3). Indeed, for source A they obtain $S_{\nu} \propto \nu^{0.72 \pm 0.07}$ and $\theta \propto$ $\nu^{-0.06 \pm 0.02}$, while for source B they obtain $S_{\nu} \propto \nu^{0.89 \pm 0.03}$ and $\theta \propto \nu^{-0.14 \pm 0.09}$. This result, if confirmed over a larger frequency range, will imply that the radial gradient model is incorrect. As an alternative explanation, we have the model of hierarchical clumps of Ignace \& Churchwell (2004), that can produce spectral indices as observed with little change in angular size with frequency. 

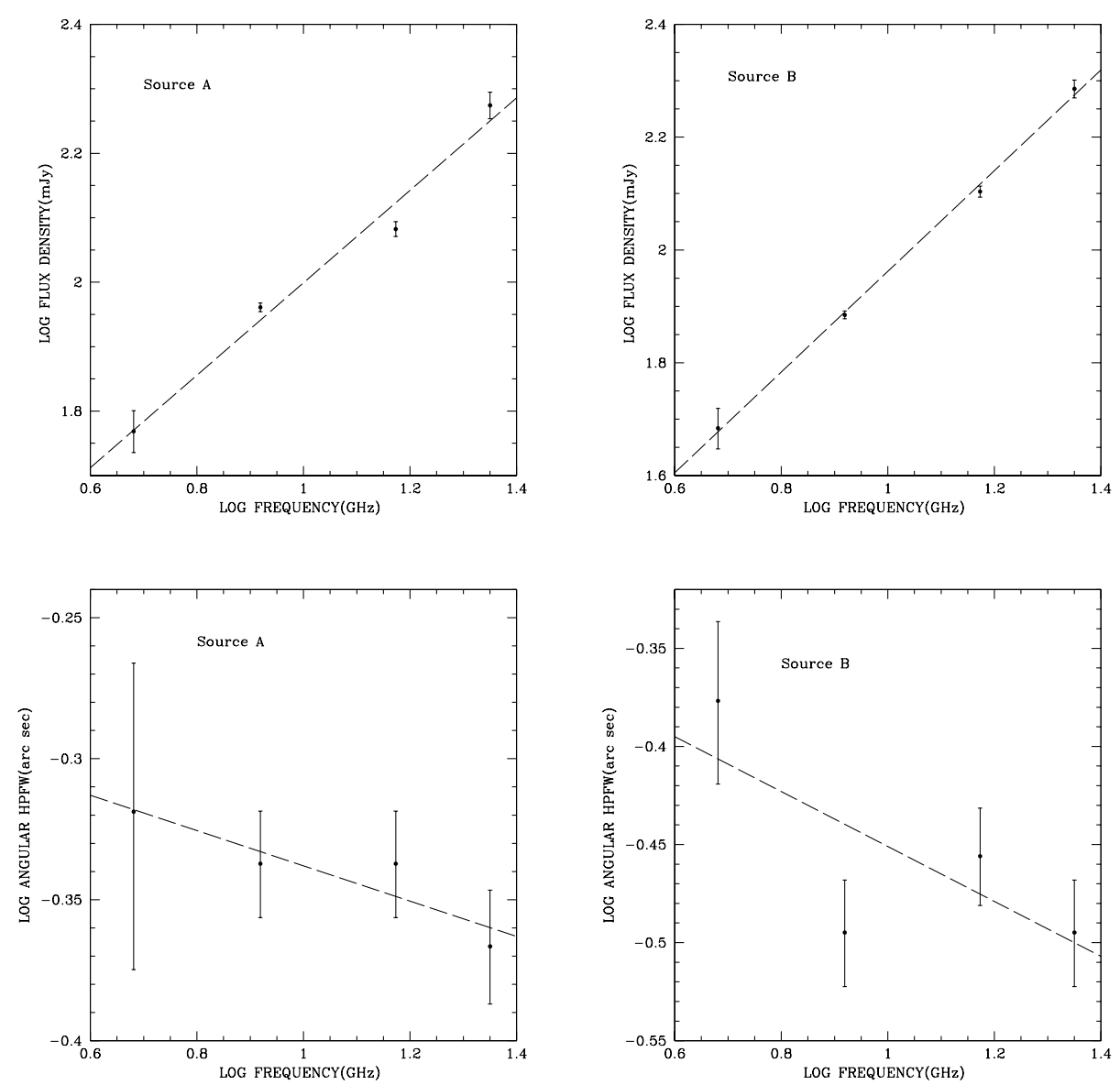

Figure 3. Flux density as a funcion of frequency (left) and deconvolved angular half power full width (right) for the hypercompact regions A (top) and B (bottom) in the G34.26+0.15 region. The dashed lines are the least squares fits to the data. While the flux densities increase approximately as $\nu^{1}$, the angular HPFWs decrease with frequency too slowly for the observations to be explained in terms of a radial gradient in the electron density. Data from Avalos et al. (2005).

\section{Changing UCHII Regions}

We usually think of UCHII regions as steady objects, with little variation along the duration of a human lifetime. However, accurate observations have shown that they are of course evolving and that change can be observed in them. We will discuss examples of UCHII regions that are expanding, varying in flux density, and finally moving in the sky.

\subsection{Expanding UCHII Regions}

Over the years, we expect the UCHII regions to show expanding motions. However, these expanding motions have been reported only in two sources: G5.89-0.39 (Acord et al. 1998) and W3(OH) (Kawamura \& Masson 1998). Acord et al. (1998) used VLA observations separated by about 5 years to detect an expansion velocity of about $35 \mathrm{~km}$ $\mathrm{s}^{-1}$ in G5.89-0.39 (see Fig. 4). In contrast to the large expansion velocity found in G5.890.39 , Kawamura \& Masson (1998) find that W3(OH) is expanding at only $3-5 \mathrm{~km} \mathrm{~s}^{-1}$. 


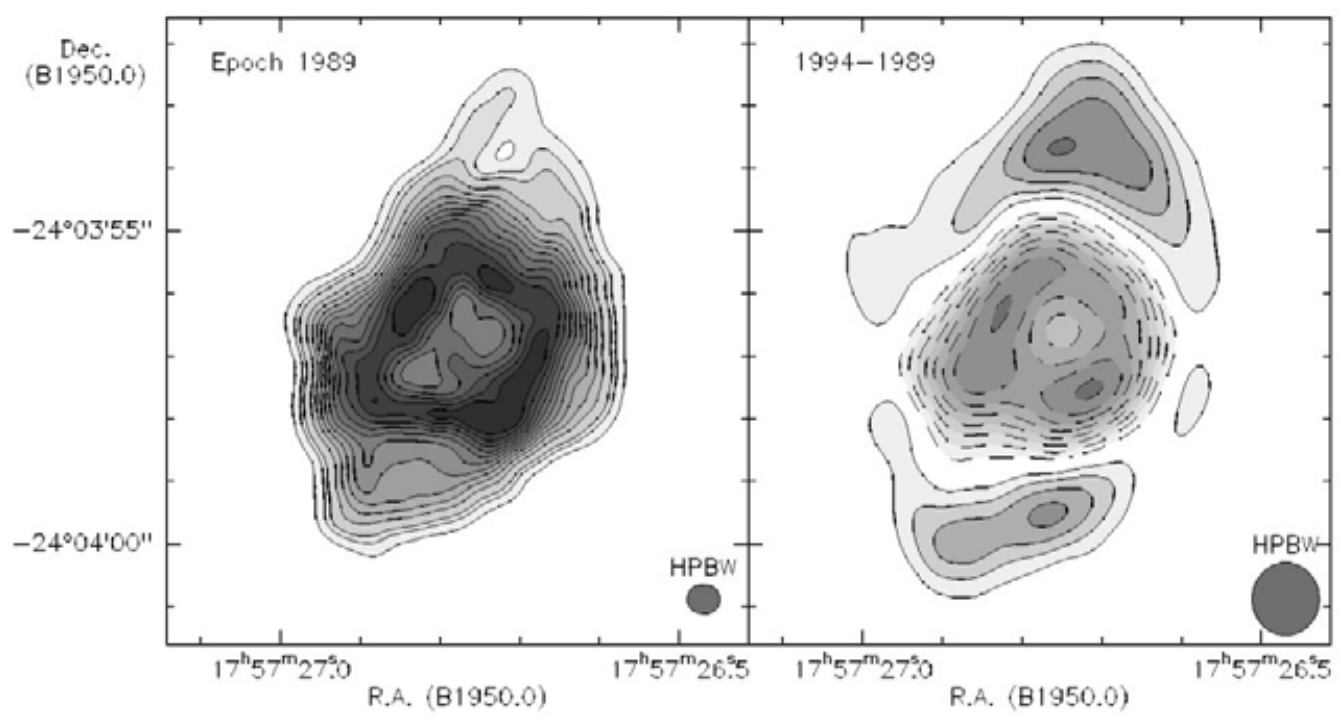

Figure 4. Left panel: the $3.6 \mathrm{~cm}$ epoch 89 map of G5.89-0.39. The contour levels are 2, 6, $10,20,30, \ldots, 110 \mathrm{mJy}^{\text {beam }}{ }^{-1}$. Right panel: the epoch 94-89 difference map of G5.89-0.39 at $3.6 \mathrm{~cm}$, showing the inner negative bowl and outer positive "ring" characteristic of expansion. The dashed contours are $-1.5,-3,-4.5, \ldots,-15 \mathrm{mJy}_{\text {beam }}{ }^{-1}$. The solid contours are $1.5,3$, $4.5,6$, and $7.5 \mathrm{mJy}^{\text {beam }}{ }^{-1}$. The half-power beamwidths (HPBWs) of the restoring beams are indicated in the lower right-hand corners. Data from Acord (1998).

The technique employed to detect these small expansive motions is the cross-calibration method developed by Masson $(1986 ; 1989)$.

Clearly, the determination of more expansion velocities (or upper limits) will be crucial to understand the "age paradox" (Churchwell 2002), that is, the fact that UCHII regions seem to remain confined for very long times. It is perplexing that the only two sources with measured expansions give values that are so different.

\subsection{An UCHII Region with Changing Flux Density}

The electron densities of the UCHII regions are quite large $\left(n_{e} \geqslant 10^{4} \mathrm{~cm}^{-3}\right)$ and if the source of ionizing radiation were shut off, the region will recombine in timescales of only a few years. Recently, Franco-Hernández \& Rodríguez (2004) presented high angular resolution ( $\left.\sim 0^{\prime \prime} 1-0{ }^{\prime \prime} 4\right)$ VLA observations at 2 (see Fig. 5 ) and $6 \mathrm{~cm}$ made in 1983, 1986, and 1995 toward the ultracompact bipolar H II region NGC 7538 IRS1. They found, at both wavelengths, clear evidence of a decrease in the emission from the lobes. This decrease, of orden $20-30 \%$, had not been observed previously in any ultracompact H II region. Franco-Hernández \& Rodríguez (2004) argue that, most likely, this decrease is due to recombination of the ionized gas in the lobes as a result of a decrease in the available ionizing photon flux. It is unclear if this decrease in the ionizing photon flux is due to an intrinsic change in the exciting star or to increased absorption of ionizing photons in the optically-thick core of the nebula.

Unfortunately, these two possibilities cannot be disentangled at present given the large free-free opacity of the core at centimeter wavelengths. Future observations above 100 $\mathrm{GHz}$ (where all the nebula is expected to be optically thin in the free-free) could show that the total flux density of the region remains constant, with the lobes decreasing and the core increasing correspondingly. If this is the case, one would conclude that the decrease observed in the lobes is due to increased absorption of ionizing photons in the 


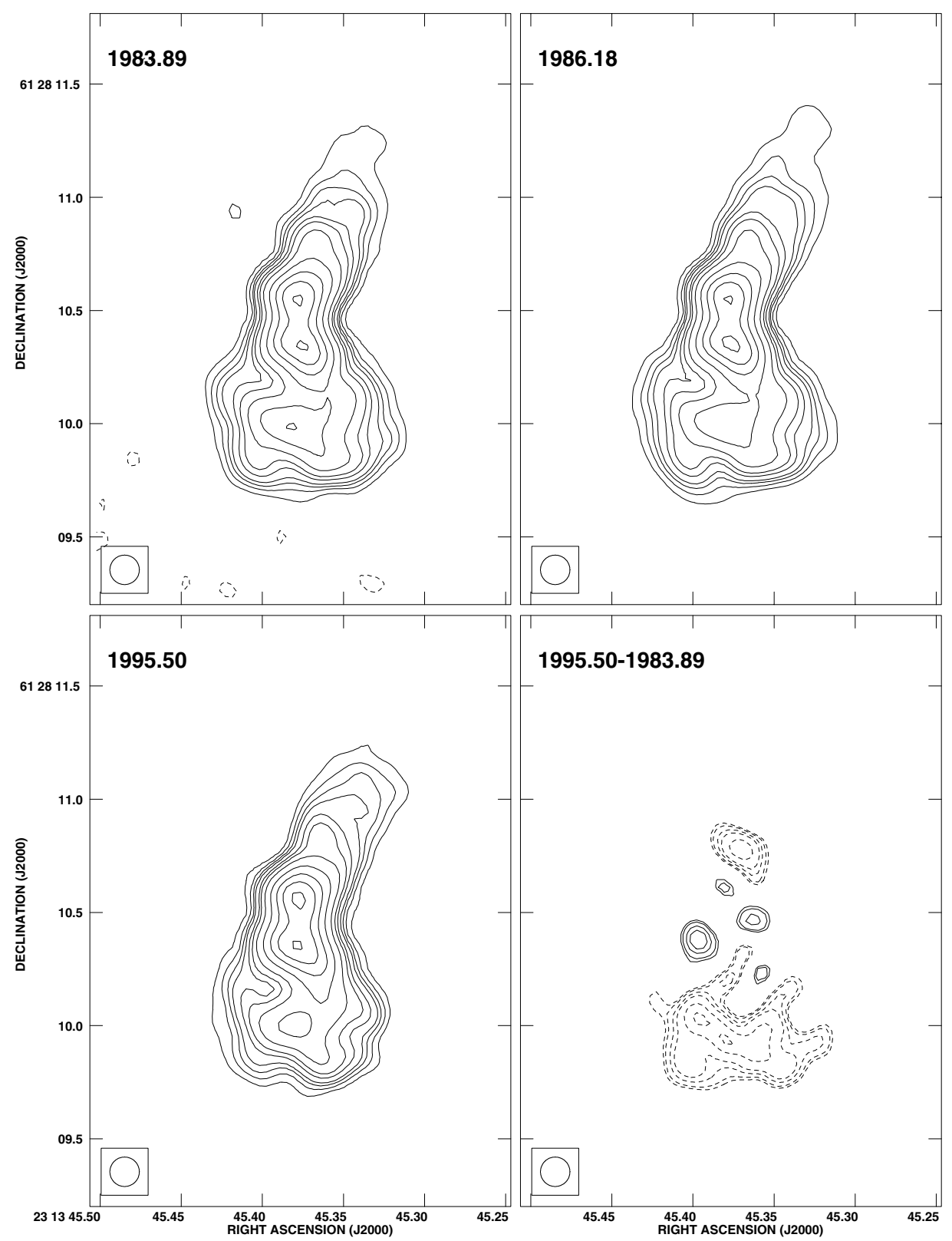

Figure 5. VLA images of NGC 7538 IRS1 at $2 \mathrm{~cm}$ for epochs 1983.89, 1986.18, and 1995.50, as well as the difference image for 1995.50-1983.89. All images are reconstructed with a circular Gaussian beam with HPBW of 0.13 . The contours for the images of the individual epochs are $-5,5,10,15,20,30,40,60,100,150,200$, and 250 times $0.1 \mathrm{mJy}^{\text {beam }^{-1}}$, the average $\mathrm{rms}$ of the three images. The contours for the difference image are $-40,-30,-25,-20,-15,-10,-8,-6$, $-5,5,6,8,10,15$, and 20 times 0.2 mJy beam $^{-1}$. The negative contours at the position of the lobes in this difference image imply a decrease in the flux density. Data from Franco-Hernández \& Rodríguez (2004). 

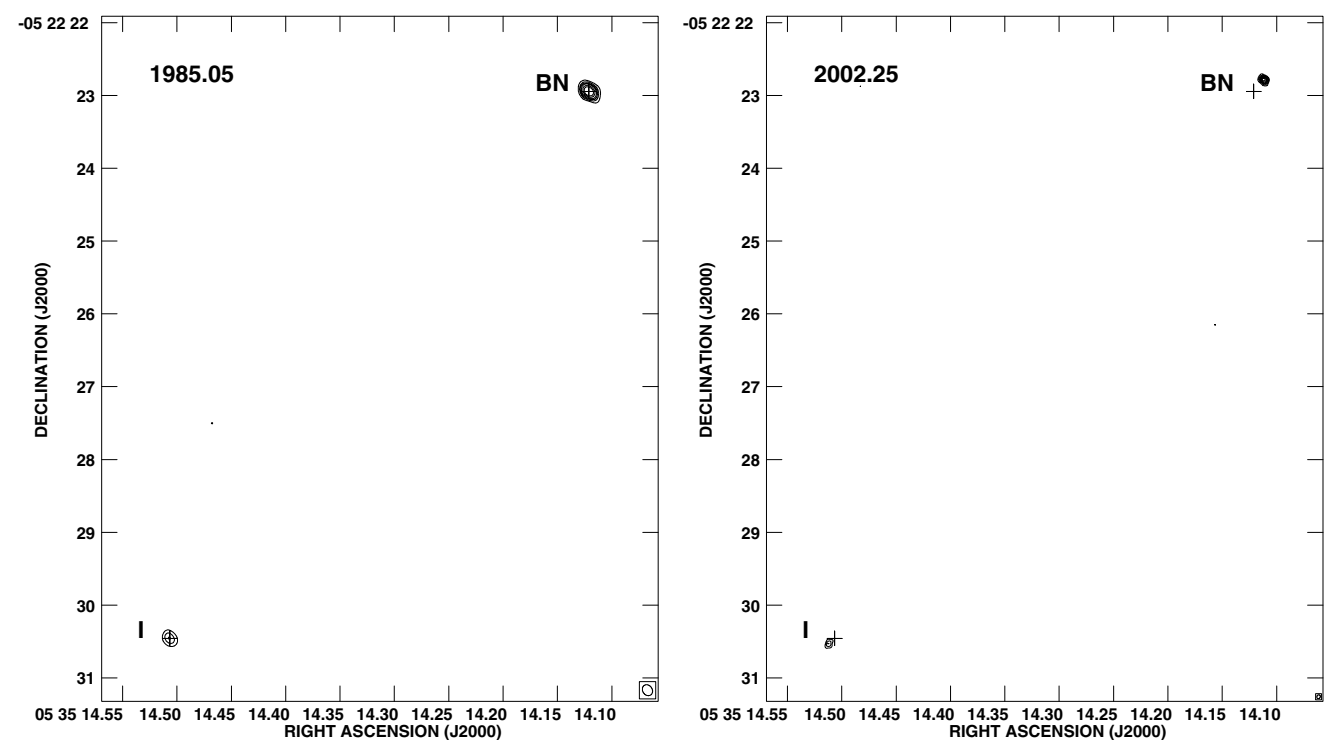

Figure 6. VLA images for 1985.05 (left; $2 \mathrm{~cm}$ ) and 2002.25 (right; $7 \mathrm{~mm}$ ) towards the BN object and the I radio source. The crosses mark the 1985.05 position of the sources. Contours are $-4,4,8,12,16,24$, and 32 times 0.13 (left) and $0.37 \mathrm{mJy}^{-1}$ beam $^{-1}$ (right), the rms of the respective images. The half power contour of the synthesized beams $\left(00^{\prime \prime} 16 \times 00^{\prime \prime} 13 ; \mathrm{PA}=32^{\circ}\right.$ for the $2 \mathrm{~cm}$ image and $0 .{ }^{\prime \prime} 06 \times 00^{\prime \prime} 05 ; \mathrm{PA}=-34^{\circ}$ for the $7 \mathrm{~mm}$ image) are shown in the bottom right corner of each panel. Note the proper motion of both sources. Data from Rodríguez et al. (2005).

core of NGC 7538 IRS1. For example, there could be increased injection of gas from the neutral torus into the surroundings of the central star, decreasing the amount of photons available for the lobes.

\subsection{Moving UCHII Regions}

There is at least one example of an UCHII region that is moving relatively fast with respect to its ambient cloud. Indeed, in the radio the BN object is detected as a UCHII that is known to be moving to the NW with a speed of several tens of $\mathrm{km} \mathrm{s}^{-1}$ (Plambeck et al. 1995). In a recent analysis of the data, Tan (2004) proposed that the BN object was ejected some 4,000 years ago by dynamical interactions in a multiple system located at $\theta^{1} \mathrm{C}$ Ori, the brightest star of the Orion Trapezium. However, absolute astrometry of the VLA data taken over the last two decades (Rodríguez et al. 2005) suggests that the radio source I (apparently a thermal jet) is also moving in the sky, receding from a point between it and the BN object (see Fig. 6)

Rodríguez et al. (2005) conclude that $\mathrm{BN}$ is moving to the $\mathrm{NW}$ at a velocity of $27 \pm 1 \mathrm{~km} \mathrm{~s}^{-1}$, while the radio source I is moving to the SE at a velocity of $12 \pm 2 \mathrm{~km} \mathrm{~s}^{-1}$. The data suggest that some 500 years ago, a multiple stellar system, formed at least by $\mathrm{BN}$ and I had a close encounter and the stars were expelled in antiparallel directions. Either BN or I have to be close binary systems for this scenario to work. It is believed that encounters in multiple stellar systems can lead to the formation of close binaries or even mergers, with eruptive outflows produced simultaneously (Bally \& Zinnecker 2005). It is very interesting that around the BN and I sources there is the well-known fast, poorly collimated OMC1 CO outflow (Kwan \& Scoville 1976), that is also detected in the form of the $\mathrm{H}_{2}$ "fingers" (Allen \& Burton 1993). This outflow system has a kinematic 
age of about 1,000 years (Doi, O'Dell, \& Hartigan 2002), but it can be argued that this timescale is more like an upper limit and that the ejection of BN and I and of the molecular gas took place in the same event 500 years ago.

\section{Conclusions}

Although we understand UCHII regions in their basic aspects, many of their characteristics remain poorly comprehended. The origin of their morphologies and their electron density structure remain controversial. We argued that time variation is a new parameter that is starting to come into the analysis and that will shed light on these and other issues. In particular, we presented examples of UCHII regions that are expanding, varying in flux density, or moving in the sky.

\section{Acknowledgements}

I acknowledge the support of CONACyT, México and DGAPA, UNAM.

\section{References}

Acord, J. M., Churchwell, E., \& Wood, D. O. S. 1998, ApJ 495, L107

Allen, D. A., \& Burton, M. G. 1993, Nature 363, 54

Avalos, M. et al. 2005, in preparation

Bally, J., \& Zinnecker, H. 2005, AJ 129, 2281

Carral, P., Kurtz, S. E., Rodríguez, L. F., Menten, K., Cantó, J., \& Arceo, R. 2002, AJ 123, 2574

Churchwell, E. 2002, ARA\&A 40, 27

De Pree, C. G., Wilner, D. J., Deblasio, J., Mercer, A. J., \& Davis, L. E. 2005, ApJ 624, L101

Doi, T., O'Dell, C. R., \& Hartigan, P. 2002, AJ 124, 445

Franco, J., Kurtz, S., Hofner, P., Testi, L., García-Segura, G., \& Martos, M. 2002, ApJ 542, L143

Franco-Hernández, R., \& Rodríguez, L. F. 2004, ApJ 604, L105

Ignace, R., \& Churchwell, E. 2004, ApJ 610, 351

Kawamura, J. H., \& Masson, C. R. 1998, ApJ 509, 270

Kim, K.-T., \& Koo, B.-C. 2003, ApJ 596, 362

Kurtz, S., Churchwell, E., \& Wood, D. O. S. 1994, ApJS 91,659

Kwan, J., \& Scoville, N. Z. 1976, ApJ 210, L39

Masson, C. R. 1986, ApJ 302, L27

Masson, C. R. 1989, ApJ 336, 294

Panagia, N., \& Felli, M. 1975, A\&\&A 39, 1

Rodríguez, L. F., Poveda, A., Lizano, S., \& Allen, C. 2005, to appear in ApJL

Tan, J. 2004, ApJ 607, L47

Wood, D. O. S., \& Churchwell, E. 1989, ApJS 69, 831 